\title{
GPS scintillations and total electron content climatology in the southern low, middle and high latitude regions
}

\author{
Luca Spogli ${ }^{1{ }^{\star}}$, Lucilla Alfonsi ${ }^{1}$, Pierre J. Cilliers ${ }^{2}$, Emilia Correia ${ }^{3}$, Giorgiana De Franceschi ${ }^{1}$, \\ Cathryn N. Mitchell ${ }^{4}$, Vincenzo Romano ${ }^{1}$, Joe Kinrade ${ }^{4}$, Miguel Angel Cabrera ${ }^{5}$
}

${ }^{1}$ Istituto Nazionale di Geofisica e Vulcanologia, Sezione Roma 2, Rome, Italy

${ }^{2}$ South African National Space Agency (SANSA), Space Science Directorate, Western Cape, South Africa

${ }^{3}$ Instituto Nacional de Pesquisas Espaciais (INPE), Centro de Rádio Astronomia e Astrofisica Mackenzie (CRAAM), São José dos Campos, Brazil

${ }^{4}$ University of Bath, Electronic and Electrical Engineering, Bath, United Kingdom

${ }^{5}$ Laboratorio de Telecomunicaciones, Universidad Nacional de Tucumán(UNT), Facultad de Ciencias Exactas y Tecnología

(FACET), Departamento de Electricidad, Electrónica y Computación, Tucumán, Argentina

\author{
Article history \\ Received October 22, 2012; accepted March 27, 2013. \\ Subject classification: \\ Scintillations, Dynamics, Solar-terrestrial interaction, Space weather, Statistical analysis.
}

\begin{abstract}
In recent years, several groups have installed high-frequency sampling receivers in the southern middle and high latitude regions, to monitor ionospheric scintillations and the total electron content (TEC) changes. Taking advantage of the archive of continuous and systematic observations of the ionosphere on L-band by means of signals from the Global Positioning System (GPS), we present the first attempt at ionospheric scintillation and TEC mapping from Latin America to Antarctica. The climatology of the area considered is derived through Ground-Based Scintillation Climatology, a method that can identify ionospheric sectors in which scintillations are more likely to occur. This study also introduces the novel ionospheric scintillation 'hot-spot' analysis. This analysis first identifies the crucial areas of the ionosphere in terms of enhanced probability of scintillation occurrence, and then it studies the seasonal variation of the main scintillation and TEC-related parameters. The results produced by this sophisticated analysis give significant indications of the spatial/temporal recurrences of plasma irregularities, which contributes to the extending of current knowledge of the mechanisms that cause scintillations, and consequently to the development of efficient tools to forecast space-weather-related ionospheric events.
\end{abstract}

\section{Introduction}

Ionospheric scintillations are driven by ionospheric irregularities. Ionospheric irregularities are commonly indicated as regions of enhanced or depleted electron density that can suddenly form in the ambient ionosphere in response to many phenomena, such as the forcing of the solar wind. Such structures vary across a very broad range of spatial scales: from hundreds of kilometers down to centimeters. Under perturbed conditions induced by the impact of solar storms on the near-Earth environment, the ionosphere can become highly turbulent, and the probability of the formation of an irregularity can significantly increase.

As trans-ionospheric signals propagate through such irregularities, they encounter gradients of electron density, which results in two main effects: group delay and phase advance, which give rise to refraction and diffraction [e.g., Yeh and Liu 1982, Kintner et al. 2009]. Moreover, as the signal keeps propagating down to the ground, small changes in the distance of propagation along the scattered ray paths cause the signal to interfere with itself. The overall effect is a sort of 'space multipath', as the distortion of the original wave front, which gives rise to a randomly modulated wave. When received at the ground, the signal can undergo rapid, random variations of amplitude and phase that are commonly known as 'ionospheric scintillation'.

When sufficiently strong, ionospheric scintillations can bedevil Global Navigation Satellite Systems (GNSS), because the intensity of the received signal might drop below the tracking threshold (amplitude scintillation) or the carrier phase might experience rapid changes, causing cycle slips that affect the phaselock loop (phase scintillation). The resilience of GNSS to space weather events is becoming one of the crucial aspects of modern society, as many technologies and social needs rely on the robustness and availability of 
precise positioning (see, e.g., Fisher and Kunches [2011] and Committee on the Societal and Economic Impacts of Severe Space Weather Events [2008]).

The features that characterize ionospheric scintillation on radio waves are modulated by a variety of different factors, such as: operating frequency, local time, season, magnetic activity, solar cycle and geographic location. In particular, as a result of the morphology of the Earth magnetic field, the magnetic poles and equator are more affected by the scintillations.

Irregularities in the high latitude region can result from patches of plasma density, as large scale plasma blobs and density troughs, the steep edges of which are unstable, so that smaller scale density structures develop along these edges. At high latitudes within the auroral oval and cusp, precipitating energetic particles produce enhanced electron densities. The fluxes of these precipitating particles are structured in space and time and create irregular structures in the ionosphere that correspond to the boundaries of the auroral oval. Within the polar cap, ionospheric irregularities are associated with patches, as discrete electron density enhancements in the $\mathrm{F}$ layer that are characterized by horizontal scales from a few hundreds to a $1000 \mathrm{~km}$, and convecting anti-sunward (see, e.g., Tsunoda [1988], and references therein). Polar patches are the main driver of scintillation within the cap [De Franceschi et al. 2008].

At low latitude, amplitude scintillations are stronger with respect to other latitudes [Basu et al. 2002]. At the magnetic equator, the $B$ field is parallel to the Earth surface and produces the so-called 'fountain effect': an ionospheric plasma movement upwards and downwards, due to the interplay among $\mathbf{E x B}$ drift, gravity and pressure gradients. This mechanism leads to the formation of the Equatorial Ionospheric Anomaly, with the minimum F-region ionization density at the magnetic equator and the maxima at the two crests, which are located roughly at $20^{\circ}$ in magnetic latitude northward and southward. At the local post-sunset hours, a pre-reversal enhancement of the eastward electric field leads to the formation of a Rayleigh-Taylor instability [e.g., Jin et al. 2008]. In this time range, the equatorial $F$ layer rises up under the action of the enhanced evening eastward electric field, and a bottom-side density gradient region develops, the equatorial spread-F, which allows the formation of ionospheric irregularities. These are mainly large electron density depletions that are elongated along the magnetic lines, and are often called 'plasma bubbles' [see, e.g., Young et al. 1984]. Recently, the development of the equatorial spread-F has been shown to also be driven by gravity waves [Abdu et al. 2009, Cabrera et al. 2010].
The investigated sector is also characterized by the South Atlantic Magnetic Anomaly (SAMA), which is a large anomaly of the Earth magnetic field that is characterized by the lowest values of the geomagnetic field intensity at the Earth surface. The SAMA allows a large particle radiation flux to come into the atmosphere [Abdu et al. 2005]. Similarly, even if less severely than in the cusp region at polar latitudes, particle precipitation in the SAMA is another source of ionospheric turbulence that can lead to scintillation, as it disturbs the thermospheric circulation in the atmosphere and alters the rates of production and recombination of the ionized species, mainly under geomagnetic storms.

In this study, the main features of the high, mid and low latitude ionosphere in response to the scintillation phenomenon on global positioning system (GPS) signals are depicted by analyzing data from a network of GPS ionospheric scintillation and total electron content (TEC) monitor (GISTM) receivers located in the southern hemisphere, between South America, the South Atlantic Ocean and Antarctica. In particular, the similarities and differences in the mechanisms that lead to scintillation among the different areas are highlighted by means of the climatological representation given by the Ground-Based Scintillation Climatology (GBSC) technique [Spogli et al. 2009, 2010, Alfonsi et al. 2011]. The novelty of this study is also the seasonal characterization of the here-defined ionospheric scintillation 'hot spots', which are presented for the first time. These hot spots are defined using the GBSC representation as the crucial areas of the ionosphere in which the probability of having scintillation is enhanced.

\section{Measurements and parameters adopted}

We have analyzed the data from a network of nine GISTM receivers that covers a very large area of the southern hemisphere, from sub-equatorial Latin America to the South Pole, across the South Atlantic Ocean. The GISTMs consist of NovAtel OEM4 dual-frequency receivers with special firmware designed to compute in near real-time the widely used amplitude $\left(S_{4}\right)$ and phase $\left(\sigma_{\Phi}\right)$ scintillation indices from the GPS L1 frequency signals, the ionospheric TEC and its changes (rate of TEC; ROT) from the GPS L1 and L2 carrier-phase signals. In this study, these quantities are evaluated using $50-\mathrm{Hz}$ sampling of the signals [Van Dierendonck et al. 1993], with average values obtained every minute.

Scintillation indices are here projected to the vertical, to account for varying geometrical effects on the measurements made at different elevation angles, as in the following formulae: 


$$
\begin{aligned}
\sigma_{\Phi}^{\text {vert }} & =\frac{\sigma_{\Phi}^{\text {slant }}}{\left(F\left(\alpha_{\text {elev }}\right)\right)^{a}}, \\
S_{4}^{\text {vert }} & =\frac{S_{4}^{\text {slant }}}{\left(F\left(\alpha_{\text {elev }}\right)\right)^{b}},
\end{aligned}
$$

where $\sigma_{\Phi}^{\text {slant }}$ and $S_{4}^{\text {slant }}$ are the indices that are directly provided by the receiver at a given elevation angle along the slant path. In Equations (1) and (2), $F\left(\alpha_{\text {elev }}\right)$ is the obliquity factor, which is defined as [Mannucci et al. 1993]:

$$
\mathrm{F}\left(\alpha_{\text {elev }}\right)=\frac{1}{\sqrt{1-\left(\frac{R_{E} \cos \alpha_{\text {elev }}}{R_{E}+H_{I P P}}\right)^{2}}},
$$

where $R_{E}$ is the Earth radius, and $H_{I P P}$ is the height of the Ionospheric Piercing Point (IPP), which is assumed to be $350 \mathrm{~km}$. According to Rino [1979a, b] and Wernik et al. [2003], and as discussed by Spogli et al. [2009] and Alfonsi et al. [2011], the exponent $a$ is assumed to be 0.5, while $b$ depends on the spectral index of the phase scintillation spectrum $p$, and on the anisotropy of the irregularities. It is not possible to assume a value that is valid for all of the ionospheric conditions that occur from equatorial to polar latitudes, although, as the statistical nature of the proposed study, different assumptions on $b$ do not meaningfully change the features depicted in the Discussion and Results given in the present study. For this reason, we use the values of Spogli et al. [2009] and we assume $p=2.6$, which corresponds to $b=0.9$.

Figure 1 illustrates the locations of the stations that constitute the network, while Table 1 gives the geo-

\begin{tabular}{lcccc}
\hline Location & ID & Owner & Latitude & Longitude \\
\hline Mario Zucchelli Station & BTN0S & INGV & $74.7^{\circ} \mathrm{S}$ & $164.1^{\circ} \mathrm{E}$ \\
Concordia Station Dome C 0 & DMCOS & INGV & $75.1^{\circ} \mathrm{S}$ & $123.3^{\circ} \mathrm{E}$ \\
Concordia Station Dome C 1 & DMC1S & INGV/BATH & $75.1^{\circ} \mathrm{S}$ & $123.3^{\circ} \mathrm{E}$ \\
San Miguel de Tucumán & TUC0S & INGV/UNT & $26.8^{\circ} \mathrm{S}$ & $65.20 \mathrm{~W}$ \\
Gough Island & GOGGS & SANSA & $40.4^{\circ} \mathrm{S}$ & $9.9^{\circ} \mathrm{W}$ \\
Marion Island & MARGS & SANSA & $46.9^{\circ} \mathrm{S}$ & $37.9^{\circ} \mathrm{E}$ \\
Comandante Ferraz Station & EACF0 & INPE & $62.1^{\circ} \mathrm{S}$ & $58.4^{\circ} \mathrm{W}$ \\
Radio Observatorio do Itapetinga & ROIOS & INPE & $23.2^{\circ} \mathrm{S}$ & $46.6^{\circ} \mathrm{W}$ \\
South Pole & SPOLE & BATH & $90^{\circ} \mathrm{S}$ & $0^{\circ} \mathrm{E}$ \\
\hline
\end{tabular}

Table 1. Summary of the receiver locations and their main features.

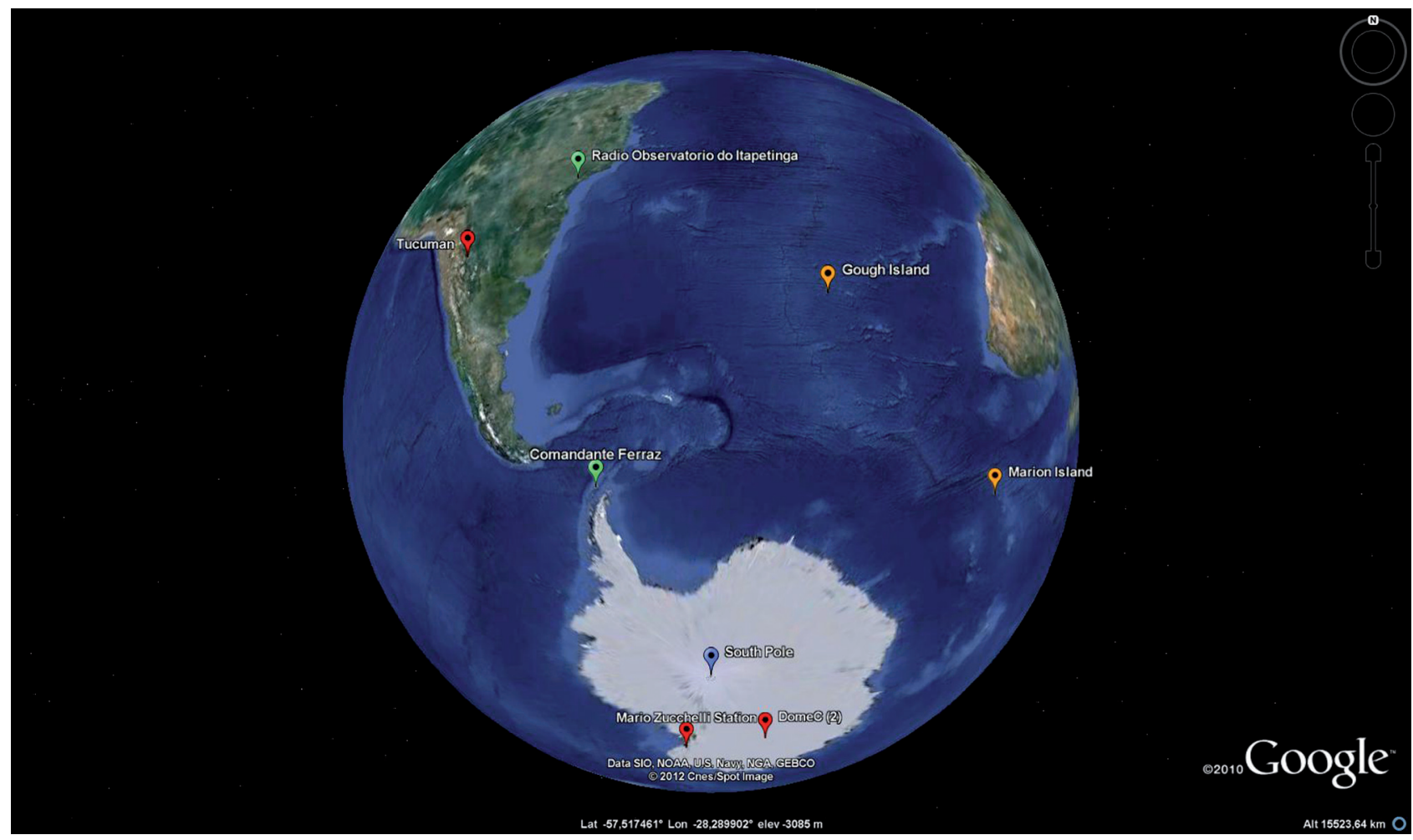

Figure 1. Location of the receivers used in this study. 


\begin{tabular}{lccccccccc}
\hline Month & $\begin{array}{c}\text { TUCOS } \\
(\%)\end{array}$ & $\begin{array}{c}\text { BTNOS } \\
(\%)\end{array}$ & $\begin{array}{c}\text { DMCOS } \\
(\%)\end{array}$ & $\begin{array}{c}\text { DMC1S } \\
(\%)\end{array}$ & $\begin{array}{c}\text { EACF0 } \\
(\%)\end{array}$ & $\begin{array}{c}\text { ROIOS } \\
(\%)\end{array}$ & $\begin{array}{c}\text { GOGGS } \\
(\%)\end{array}$ & $\begin{array}{c}\text { MARGS } \\
(\%)\end{array}$ & $\begin{array}{c}\text { SPOLE } \\
(\%)\end{array}$ \\
\hline Jan & 58 & 100 & 100 & 100 & 97 & 94 & 100 & 0 & 68 \\
Feb & 86 & 100 & 100 & 100 & 100 & 86 & 100 & 4 & 100 \\
Mar & 100 & 100 & 100 & 100 & 97 & 100 & 45 & 45 & 100 \\
Apr & 100 & 100 & 100 & 100 & 100 & 97 & 0 & 90 & 100 \\
May & 100 & 100 & 100 & 100 & 100 & 100 & 0 & 90 & 100 \\
Jun & 100 & 100 & 100 & 100 & 100 & 100 & 0 & 73 & 100 \\
Jul & 100 & 100 & 100 & 87 & 100 & 100 & 16 & 100 & 100 \\
Aug & 100 & 100 & 100 & 100 & 94 & 100 & 100 & 90 & 100 \\
Sep & 100 & 100 & 100 & 100 & 100 & 100 & 100 & 100 & 100 \\
Oct & 71 & 100 & 100 & 100 & 100 & 100 & 100 & 52 & 100 \\
Nov & 7 & 70 & 100 & 47 & 100 & 100 & 100 & 0 & 90 \\
Dec & 0 & 0 & 100 & 87 & 97 & 90 & 97 & 0 & 97 \\
\hline
\end{tabular}

Table 2. Days of available data in each month and for each receiver, expressed as percentages.

graphic coordinates, the station identifiers (IDs), and the owners. Data were acquired for all of 2011, during the beginning of the rising phase of the approaching solar maximum (minimum average sunspot number $=18.8$; maximum average sunspot number $=96.7$ ). To show the consistency of the data to be simultaneously analyzed over a whole year, Figure 2 shows the percentages of days of available data for 2011 for each receiver. The overall availability is good and is well above $70 \%$, except for the two stations located in the South Atlantic, Gough Island (ID: GOGGS, 63\%) and Marion Island (ID: MARGS, 70\%). As this study also presents an investigation dedicated to seasonal variations of the scintillation features, it is important to identify whether data gaps are present in particular months. Table 2 summarizes the percentages of the days of available data in each month and for each receiver. For MARGS, the main gaps were from January to February and November to December, and for GOGGS, from April to July. Other meaningful data gaps are the almost total lack of data for Tucumán (ID: TUC0S, Argentina) from November

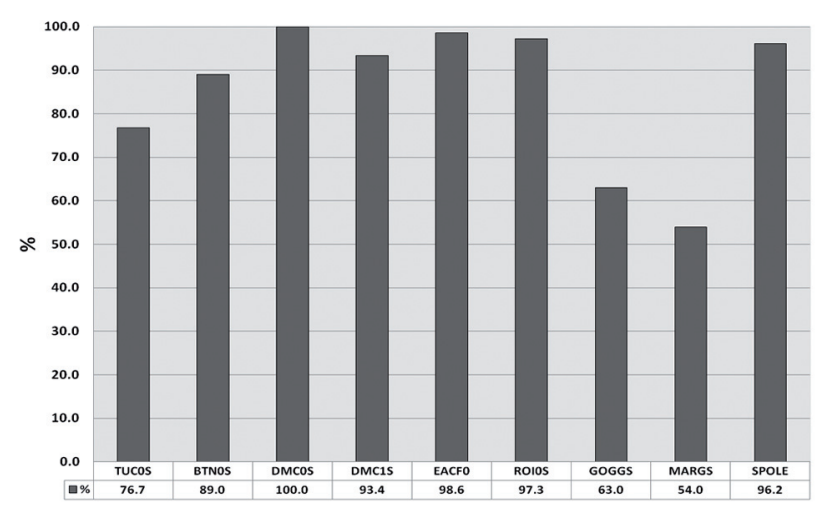

Figure 2. Percentage of days of available data in 2011 for each receiver. to December, and the total unavailability of the Mario Zucchelli station (ID: BTNOS, Antarctica) in December.

To show the coverage of the network, Figure 3 presents the combined field of view, obtained by considering all of the available satellite passes and applying an elevation angle threshold of $20^{\circ}$, which is a commonly accepted threshold to remove observations at low angles at which the multipath of the rays from terrestrial structures can mimic ionospheric scintillation. This threshold is hereafter applied to all of the data analyzed. From Figure 3 it is evident that the network covers almost all of the geographic latitudes and longitudinal sectors in the Latin America/ South Atlantic/ Antarctica area. This is also confirmed by Figure 4, which shows the distribution of the latitude by rejecting only low elevation angles (Figure 4, red). The single receiver sub-distributions are evident with 8 peaks, with only Concordia station Dome C 0 (ID: DMCOS) and Concordia station Dome C 1 (ID: DMC1S) superimposed, while they almost monotonically fade one into the other with continuity. The blue distribution in Figure 4 is obtained by transforming the geographic coordinates into altitude-adjusted corrected geomagnetic latitude (AACGM, Mlat) [Baker and Wing 1989]: the altitude considered is $H_{I P P}=350 \mathrm{~km}$, which is assumed to be the IPP. This transformation 'shrinks' the distribution into three main magnetic latitude regions:

(i) Low latitude region, from about $-28^{\circ} \mathrm{N}$ to $-15^{\circ} \mathrm{N}$ Mlat, to which TUCOS station and Radio Observatorio do Itapetinga (ID: ROIOS) contribute;

(ii) Mid latitude region, from about $-58^{\circ} \mathrm{N}$ to $-38^{\circ} \mathrm{N}$ Mlat, to which GOGGS, MARGS and Comandante Ferraz (ID: EACF0) stations contribute;

(iii) High latitude region, from about $-90^{\circ} \mathrm{N}$ to $-68^{\circ} \mathrm{N}$ Mlat, to which DMC0S, DMC1S, BTNOS and 


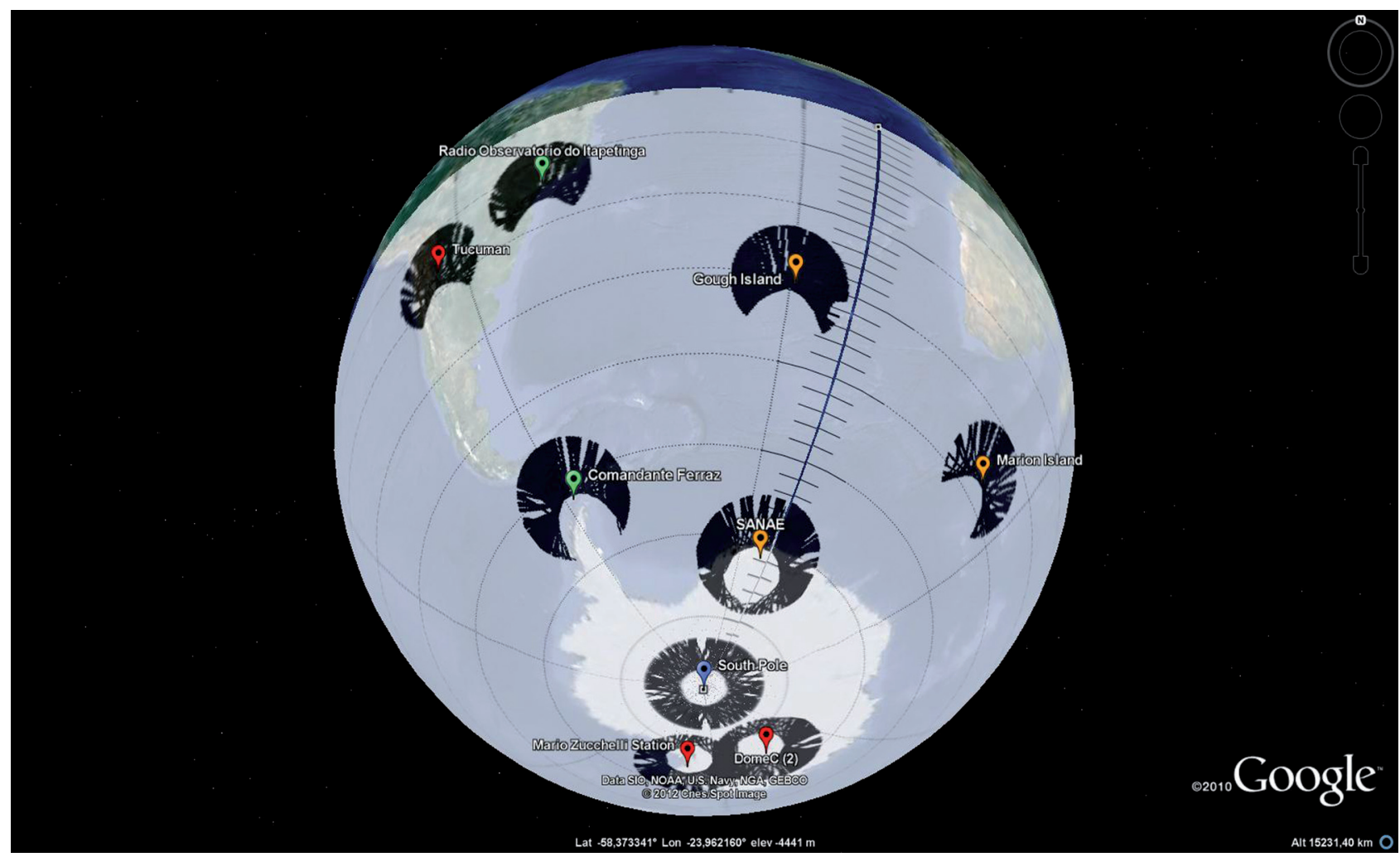

Figure 3. Field of view spanned by the network, obtained by considering all of the data available and applying an elevation angle threshold of $20^{\circ}$.

South Pole (ID: SPOLE) stations contribute.

The ranges of magnetic latitudes covered by each receiver are summarized in Table 3.

\section{Methods}

The data were analyzed using the GBSC technique, which was recently developed by the Istituto Nazionale di Geofisica e Vulcanologia (INGV). GBSC has been shown to be very promising for the identification of areas of the ionosphere in which scintillation is more likely to occur [Spogli et al. 2009, 2010], and for assessing the general recurrent features of the dynamics and temporal evolution of ionospheric irregularities, catching the correspondences with scintillation occurrence [Alfonsi et al. 2011].

This technique produces maps of the percentage occurrence and/or mean and standard deviation of scintillation indices, the TEC, the signal-to-noise ratio, and other parameters that are defined in a bi-dimensional coordinates system (geographic coordinates, geomagnetic coordinates, horizontal coordinates, time), each of which can be selected on demand.

The percentage occurrence $O$ is evaluated in each bin of the map and is defined as:

$$
O=\frac{N_{t h r}}{N_{t o t}}
$$

where $N_{t h r}$ is the number of data points that correspond to the investigated quantity above the threshold, and $N_{\text {tot }}$ is the total number of data points in the selected bin. In the present study, we considered the amplitude and phase scintillation events as those for which $S_{4}$ and $\sigma_{\Phi}$ were above 0.25 radians and 0.25 radians respectively, which are considered as moderate/ strong scintillation thresholds. To remove the contribution of bins with poor statistics that might affect the occurrence estimation, the selected accuracy is set at 5\% (see Taylor [1997] and Spogli et al. [2009] for the application to GBSC).

The climatological representation given by GBSC is here supported by the newly introduced ionospheric scintillation hot-spot analysis, which starts from the outcomes of the GBSC, to define crucial areas of the ionosphere from the scintillation point of view. After the identification of these hot spots, the seasonal vari-

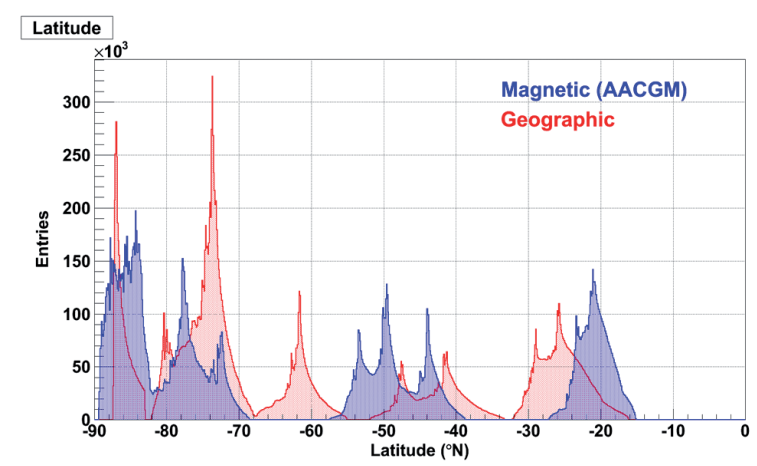

Figure 4. Distribution of geographic latitude (red) and Altitude Adjusted Corrected Geomagnetic Latitude (AACGM) at the altitude of $350 \mathrm{~km}$ (blue) for the considered period. 

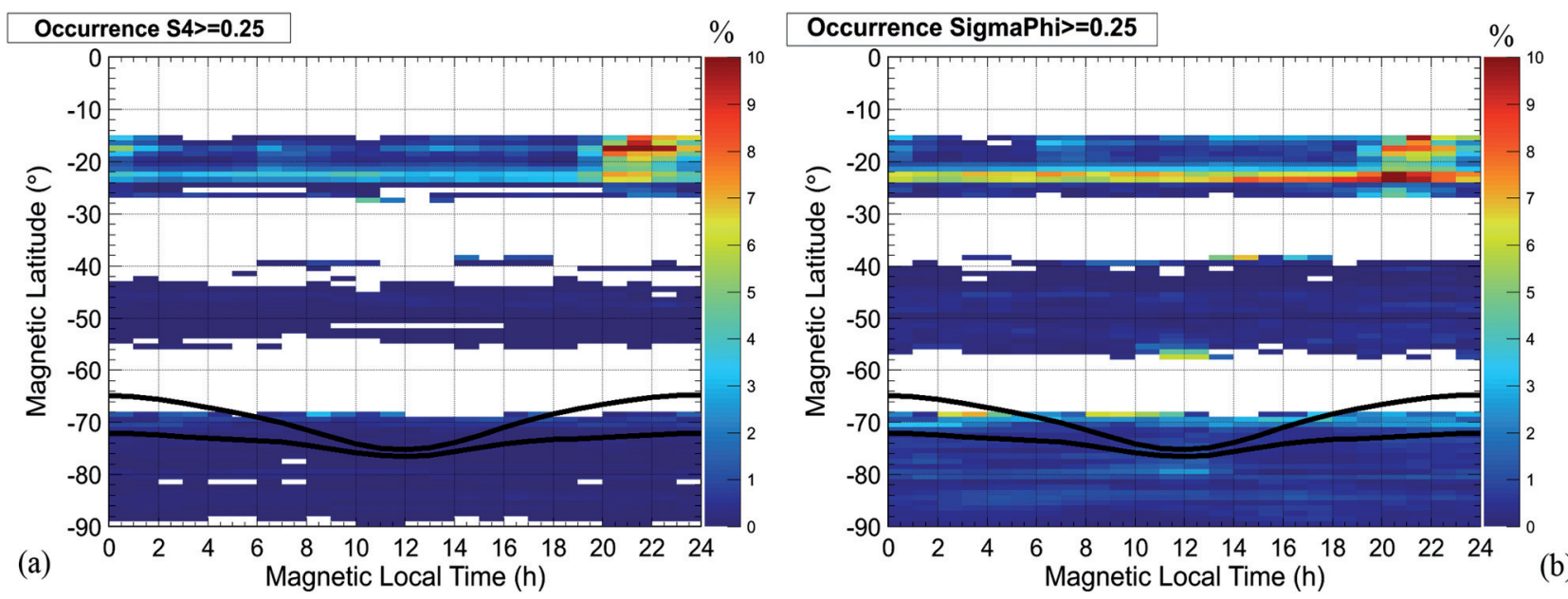

Figure 5. Maps of $S_{4}$ (a) and $\sigma_{\Phi}$ (b) percentages of occurrence above the moderate/ strong scintillation threshold. The black curve is the modeled Feldstein oval for IQ $=0$.

ations of the scintillation indices and the ROT mean and standard deviation are shown.

\section{Results}

\subsection{Climatolgical representation through Ground-Based} Scintillation Climatology

Figure 5 shows the maps of the $S_{4}(\mathrm{a})$ and $\sigma_{\Phi}$ (b) percentages of occurrence in Mlat and magnetic local time (MLT). We superimposed the position of the Feldstein auroral oval [Feldstein 1963, Holzworth and Meng 1975] for the lowest level of magnetic activity (IQ = 0) onto the scintillation occurrence maps. We chose IQ = 0 , which represents a quiet ionosphere, aware that it could give only an average representation of the position of the auroral oval boundaries, which could be located more equator-wards under disturbed geomagnetic conditions. Hereafter, we refer to this oval as the quiet oval. Even if the AACGM latitudes are more suitable as a framework for the high latitude ionosphere studies, they also highlight the main features of scintillation at mid and low latitudes, as it is evident in Figure 5. The maps of Figure 5 show the differences in the impact of scintillation at high, mid and low latitudes. Mid latitudes are characterized by very small scintillation activity, both in amplitude and phase, with occurrence generally below $1 \%$ for all Mlats and MLTs. There are some bins with enhanced probability only for phase scintillation, which are mainly located along the poleward boundaries of the mid latitude region. Moreover, phase scintillation is present in almost all of the bins, while amplitude scintillation is absent in several others.

Amplitude scintillation at high latitude is of the same order of magnitude as at mid latitude $(\approx 1 \%)$, while phase scintillation is enhanced near the boundaries of the auroral oval, at the cusp, and in the polar cap $\left(\right.$ Mlat $\left.<-80^{\circ} \mathrm{N}\right)$.
At about $-70^{\circ} \mathrm{N}$, the phase scintillation occurrence is larger in the post-midnight sector than in the pre-midnight sector, while due to the presence of the polar patches, the scintillation in the polar cap is asymmetric around midnight and favors the dawn sector. The phase scintillation enhancement is in the dawn sector (Figure 5b); i.e., the opposite of what was found in the northern hemisphere by Spogli et al. [2009], who reported a pre-midnight enhancement of phase scintillation occurrence induced by the polar cap patches. This can be explained by the opposite circulation direction of the convection cells inside the caps of the northern and southern polar regions.

In Spogli et al. [2009], the asymmetry among premidnight and post-midnight sectors at auroral latitudes was claimed to be due to the TEC distribution that was driven by the high latitude trough position around noon in the northern hemisphere, which indicates the presence of large gradients in the electron concentration. In the southern hemisphere, the asymmetry favors the post-midnight sector, which is vice versa with respect to the northern hemisphere.

In the low latitude region (Figure 5), two main areas show evidence of percentage occurrences of $6 \%$ to $10 \%$. One is well defined in the MLT range from 1900 to 0200 , which appears to be due to the ionospheric irregularities that develop during the post-sunset local hours (at the equator, magnetic and local time are not significantly different). The other is located in a band at around $-23^{\circ} \mathrm{N}$ and has only a small dependence on time. It is present on both the amplitude and phase maps, even if it leads to a larger occurrence in the phase maps. The concurrence of amplitude and phase scintillation suggests that there is a prevalence of irregularities that have a scale size below the Fresnel scale ( $\approx 250 \mathrm{~m}$ for $\mathrm{L}_{1}$ ). Moreover, the independence from time reinforces the idea of a source of scintillation that is independent of the daily variability. 

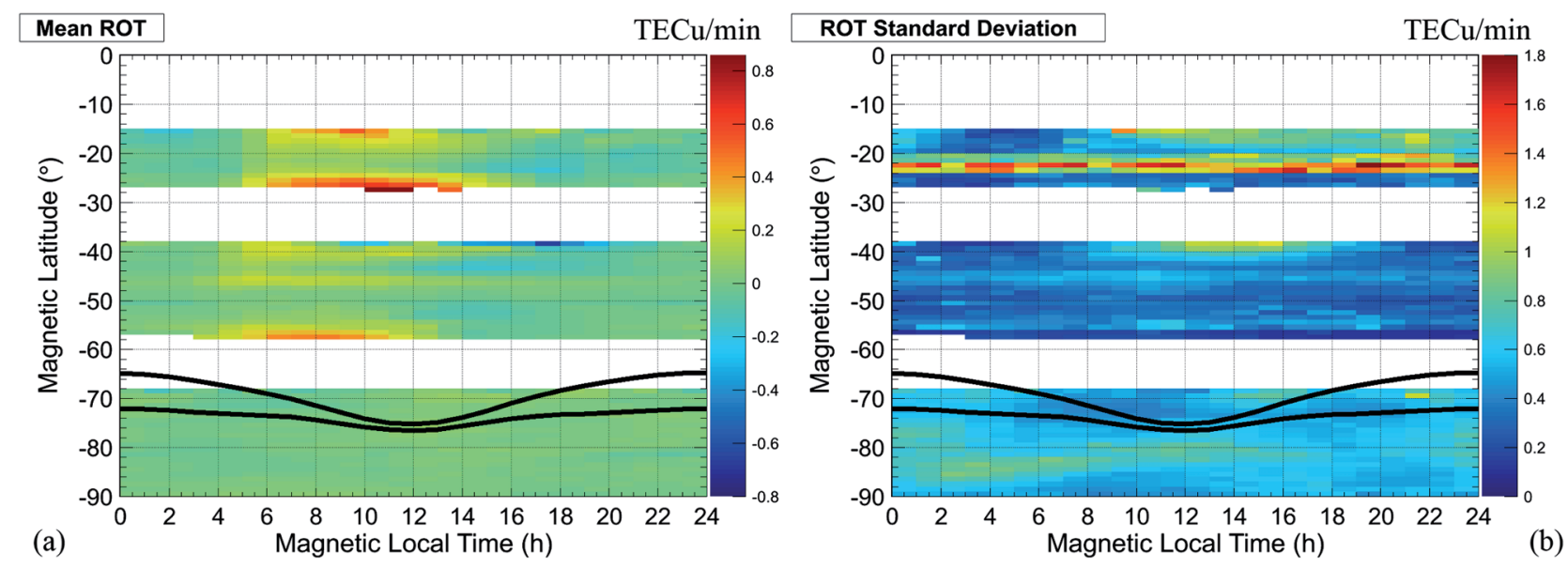

Figure 6. Maps of mean (a) and standard deviation (b) of ROT. The black curve is the modeled Feldstein oval for IQ $=0$.

A possible source of such irregularities would be the structuring imposed on the ionosphere within the SAMA by the precipitating particles.

To help with the understanding of the scale sizes involved and their correspondence with scintillation occurrence, maps of the ROT mean $(<$ ROT $>$ ) and standard deviation (ROT_SD), analogous to those in Figure 5, are shown in Figure 6. Table 3 of Alfonsi et al. [2011] reported the expected relationships between the $\mid<$ ROT $>\mid$ and ROT_SD permutations, the associated irregularities, scale sizes, and scintillation phase and/or amplitude occurrence, which are useful also for the purpose of this study. According to Table 3 of Alfonsi et al. [2011], at low latitudes the narrow band of amplitude and phase scintillation corresponds to small values of $\mid\langle$ ROT $\rangle \mid$ and large values of ROT_SD, indicating the presence of several scale sizes simultaneously induced by particle precipitation within the SAMA. The same configuration of the SAMA is found for post sunset scintillation: low $\mid<$ ROT $>\mid$ and large ROT_SD, again providing evidence of the presence of several scale sizes being active for scintillation, but here

\begin{tabular}{lcc}
\hline ID & $\begin{array}{c}\text { Mlat min } \\
\left({ }^{\circ} \mathrm{N}\right)\end{array}$ & $\begin{array}{c}\text { Mlat max } \\
\left({ }^{\circ} \mathrm{N}\right)\end{array}$ \\
\hline TUC0S & -24.5 & -15.5 \\
ROI0S & -27 & -17 \\
GOGGS & -47.5 & -39 \\
EACF0 & -55.5 & -44 \\
MARGS & -57.5 & -48 \\
SPOLE & -81.5 & -68.5 \\
BTN0S & -87 & -73.5 \\
DMC0 & -89.5 & -82.5 \\
DMC1 & -89.5 & -82.5 \\
\hline
\end{tabular}

Table 3. Ranges of magnetic latitudes covered by each receiver. concentrated in a well-defined time range.

The mid latitude region is mainly characterized by low values of both $\mid<$ ROT $>\mid$ and ROT_SD, except for a few bins equator-wards and polewards, that would need an enlargement of the field of view to be interpreted. This ROT configuration does not allow the definition of a clear scintillation scenario.

In the high latitude region, the phase scintillation occurrence follows the ROT_SD pattern, which indicates larger values mainly near the cusp, the post-midnight polar cap, and corresponding to the boundaries of the auroral oval, while the $\mid<$ ROT $>\mid$ values are generally low. According to Table 3 of Alfonsi et al. [2011], this situation is related to a possible presence of all scales, but in our case, it leads only to phase scintillation, which indicates the sole presence of scale sizes above the Fresnel distance.

\subsection{Ionospheric scintillation 'hot-spot' analysis}

The climatological picture given by the GBSC allows a general and simultaneous overview of the crucial areas of the ionosphere in which scintillation is more likely to occur: the 'hot spots'. Moreover, the choice of representing the occurrence as the AACGM is effective in depicting it. Starting from this consideration, we decided to introduce here a new analysis, in which the scintillation hot spots are first identified and defined, and then studied with respect to their seasonal variation. The analysis moves towards a deeper understanding of the relationships between the scintillation parameters and the structure and variability of the ionospheric irregularities.

The starting point of the hot-spot analysis is the map of the $\sigma_{\Phi}$ percentage of occurrence above the weak scintillation threshold (0.1 radians), as reported in Figure 7. The weak scintillation threshold is chosen here to include every meaningful bin in the hot-spot definition. The definition is based upon GBSC maps of 


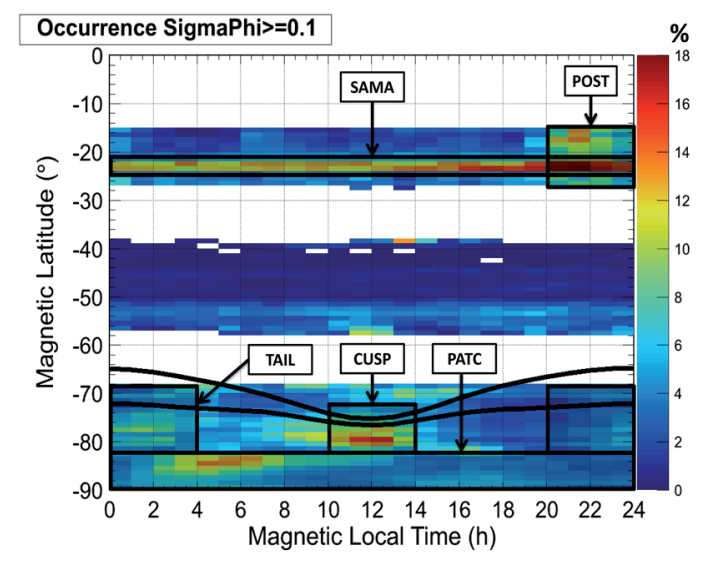

Figure 7. Map of $\sigma_{\Phi}$ percentage of occurrence above the weak scintillation threshold ( 0.1 radians). The black curve is the modeled Feldstein oval for IQ $=0$, while the black boxes define the ionospheric hot spots.

$\sigma_{\Phi}$ percentages, because at low latitudes the scintillation patterns of $S_{4}$ and $\sigma_{\Phi}$ are similar, so the hot spots there are the same. On the contrary, at high latitude, the phase scintillation occurrence identifies larger areas of enhanced scintillation probability with respect to the $S_{4}$ maps. As a consequence, the hot spots defined through phase scintillation occurrence allow us to take into account areas that are not highlighted by amplitude scintillation occurrence. In Figure 7, the quiet Feldstein oval is still present and the black boxes define the ionospheric hot spot, together with the following four-letter identifiers:

- POST, in which the scintillation is supposed to be mainly due to the post-sunset irregularities development;

- SAMA, in which the scintillation is supposed to be mainly due to irregularities induced by particle precipitation in the SAMA;

- CUSP, in which the scintillation is supposed to be mainly due to irregularities induced by direct particle precipitation at the cusp;
- TAIL, in which the scintillation is supposed to be mainly due to irregularities induced by the reconnection from the magnetotail at around magnetic noon;

- PATC, in which the scintillation is supposed to be mainly due to the TEC patches moving across the polar cap.

The definition in terms of Mlat and MLT ranges, together with the stations contributing to the analysis of the given hot spot, is summarized in Table 4. In the present study we decided not to include the mid latitude region, as scintillation occurrence is scarce with respect to the other regions.

The main outcome of the hot-spot analysis is shown in Figure 8, where the percentage occurrence above the moderate/ strong threshold for $\sigma_{\Phi}$ (Figure $8 \mathrm{a}$ ) and $S_{4}$ (Figure $8 \mathrm{~b}$ ), and of the ROT mean (Figure 8c) and standard deviation (Figure 8d) are plotted as functions of the months of 2011, and are sorted for the different hot spots. The seasonal variation also shows evidence of both phase and amplitude scintillation at low latitudes (POST, SAMA) which is of the same order of magnitude or stronger than at other latitudes.

POST presents two enhancements: the weaker one in March $(\approx 5 \%)$ and a higher one in November $(\approx 30 \%)$. This confirms what is expected with the intensification of pre-reversal at equinoxes, which is stronger in the austral spring, and in the austral summer [e.g., Fejer et al. 1989, 1991]. The enhancement observed in March was probably due to the sole equinoctial enhancement, but the peak in November was probably due to a combined effect of the spring equinox and the austral summer. The SAMA has a similar behavior, even if the effect due to the equinoxes is less evident, which possibly indicates a stronger seasonal dependence of the effects during the winter/ summer.

As far as high latitude hot spots are concerned (CUSP, PATC and TAIL), an expansion of part of Fig-

\begin{tabular}{|c|c|c|c|c|}
\hline $\begin{array}{l}\text { Ionospheric } \\
\text { hot-spot identifier }\end{array}$ & Phenomenon & $\begin{array}{l}\text { MLAT range } \\
\left({ }^{\circ} \mathrm{N} ;{ }^{\circ} \mathrm{E}\right)\end{array}$ & $\begin{array}{c}\text { MLT range } \\
(\mathrm{h})\end{array}$ & Stations involved \\
\hline POST & $\begin{array}{l}\text { Post-sunset } \\
\text { at low latitudes }\end{array}$ & $(-25 ;-15)$ & $(20 ; 24)$ & TUCoS, ROIOS \\
\hline SAMA & $\begin{array}{c}\text { Particle precipitation } \\
\text { due to SAMA }\end{array}$ & $(-24 ;-22)$ & $(0 ; 24)$ & TUCOS, ROIOS \\
\hline CUSP & $\begin{array}{l}\text { Particle precipitation } \\
\text { due to the polar cusp }\end{array}$ & $(-82 ;-74)$ & $(10 ; 14)$ & BTNOS, SPOLE \\
\hline TAIL & $\begin{array}{l}\text { Reconnection } \\
\text { from the magnetotail }\end{array}$ & $(-82 ;-68)$ & $(20 ; 4)$ & BTNOS, SPOLE \\
\hline PATC & Polar cap patches & $(-90 ;-82)$ & $(0 ; 24)$ & BTN0S, DMC0S, DMC1S \\
\hline
\end{tabular}

Table 4. Ionospheric scintillation hot spots defined in terms of the MLT and Mlat ranges. 


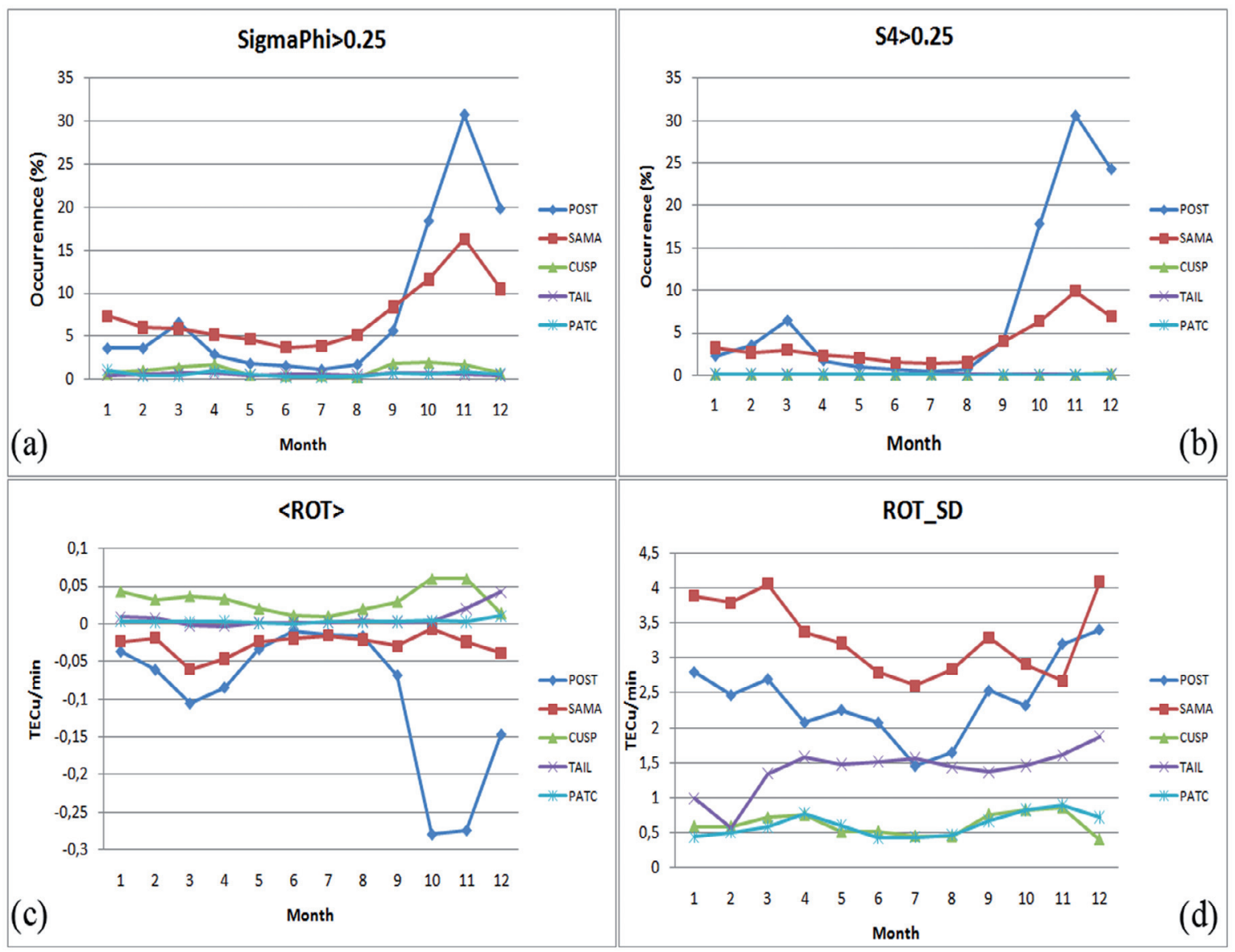

Figure 8. Seasonal variation of the percentage occurrence above the moderate/ strong threshold for $\sigma_{\Phi}(\mathrm{a})$ and $S_{4}(\mathrm{~b})$, and of the ROT mean (c) and standard deviation (d), sorted according to the different ionospheric scintillation hot spots.

ure 8a is shown in Figure 9. The main source of scintillation at high latitudes is the direct particle precipitation in the CUSP region, the peaks of which are in the equinoxes $(\approx 2 \%)$. Also TAIL and PATC present enhancements at the equinoxes, and this is because during the equinoxes the alignment between the geomagnetic field at its contact region with the interplanetary magnetic field is optimal for the merging between heliomagnetic field lines and geomagnetic field lines if they are oriented in opposite directions. The Earth magnetopause then has an optimum 'capture cross-section' to connect with a southward-oriented interplanetary magnetic field. Under these conditions, plasma from the solar wind can best enter the magnetopause and induce particle precipitation from both the cusp and tail, and can cause ionospheric irregularities.

With reference to the ROT features, Figure $8 \mathrm{c}$ shows how the low latitude hot spots, POST and SAMA, are characterized by negative values of $\langle$ ROT $>$, while the high latitude hot spots show positive $<$ ROT $>$. This confirms what is expected, because the low latitude ionosphere is characterized by the presence of plasma bubbles, which are large TEC depletions. The largest values of $<$ ROT $>$ are found in POST at the equinox and austral summer. Besides POST, only CUSP and SAMA pres- ent the same kind of seasonality at equinoxes, while PATC and TAIL have a small enhancement in December (the austral summer). Figure $8 \mathrm{~d}$ illustrates that the larger values of the ROT standard deviation correspond to the SAMA and POST regions. Two relative maxima in March and September are present, together with an increase in November and December. The ROT_SD values are associated with the intrinsic variability of the ROT: large values indicate that TEC gradients occur

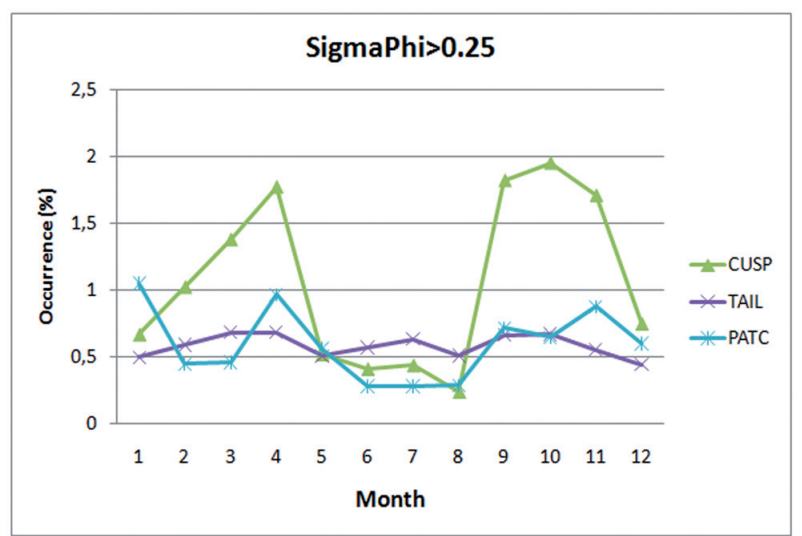

Figure 9. Seasonal variation of the percentage occurrence above the moderate/ strong threshold for $\sigma_{\Phi}$ sorted according to the ionospheric hot spots at high latitudes. 
over a wide scale range. Combining the ROT mean and standard deviation seasonality for POST and SAMA we obtain those of both phase and amplitude scintillation. Indeed, according to Table 3 of Alfonsi et al. [2011], when both ROT and ROT_SD are large, irregularities on all scales are active and produce phase and amplitude scintillation: here it appears that the larger the $\mid<$ ROT $>\mid$, the larger the scintillation occurrence, reinforced by the ROT_SD behavior.

For high latitudes, in Table 3 of Alfonsi et al. [2011], the $S_{4}$ cannot be predicted in two cases:

1. With high $\mid<$ ROT $>\mid$ and low ROT_SD, where there is a predominance of kilometer-scale structures that can cause only phase scintillation.

2. With low $\mid<$ ROT $>\mid$ and low ROT_SD, in which no prediction can be made.

Case 2 is the case of PATC, and nothing can be inferred from this variability, as it is the case in which the information coming from ROT allows nothing to be inferred about the related scintillation activity. The CUSP case is Case 1, and indeed there is no meaningful amplitude scintillation, while the phase scintillation closely follows the $<$ ROT $>$ seasonality. The case of TAIL is characterized by low ROT_SD values and larger values of $<$ ROT $>$ with respect to CUSP and PATC, but lower with respect to SAMA and POST. This is an intermediate situation in which the table does not allow an unambiguous interpretation.

\section{Conclusions}

By using the strength of the scientific collaboration within the SCAR expert group known as GRAPE, data from an extensive network of special GPS receivers for scintillation studies are analyzed here and presented through a climatological representation. The mapping covers a wide area of the southern hemisphere, from Latin America to Antarctica, across the South Atlantic Ocean.

The climatology of the GPS scintillation and TEC data is derived through the GBSC, and for the first time, the ionospheric scintillation hot-spot analysis is introduced here. The hot spots are defined by means of the GBSC representation of the phase scintillation patterns, as the crucial areas of the ionosphere in which the probability of having scintillation is enhanced.

In particular, the similarities and differences in the mechanisms leading to scintillation across the different areas is highlighted through the correspondence between the TEC variability, expressed in term of the statistical parameters of ROT, and scintillation, in term of percentage occurrence of $S_{4}$ and $\sigma_{\Phi}$ above the moderate/ strong scattering regime.

The GBSC maps in Mlats and MLT allow a picture to be drawn in which the stronger impact of scintillation, both in amplitude and phase, is found to be at equatorial latitudes and driven by two phenomena: 1) the Rayleigh-Taylor instability, which leads to plasma bubbles in the local post-sunset; and 2) the irregularities induced by particle precipitation due to SAMA. The two lead to similar occurrence of scintillation, but, while the former occurs in a specific time interval, the latter has almost no dependence on time. Mid latitudes are characterized by very small phase and amplitude scintillation activity, with very few regions characterized by enhanced probability of moderate to strong scintillation. At high latitude, it has been found that phase scintillation is enhanced along the boundaries of the auroral oval, at the cusp, and in the polar cap, with some asymmetries in the distribution due to the dynamics of the polar patches and to the average position of the high latitude trough.

The corresponding maps of $<$ ROT $>$ and ROT_SD allow the interpretation of scintillation occurrence in terms of TEC variability, and consequently, ionospheric irregularity scale sizes. The simultaneous presence of different scale sizes helps in the explanation of the scintillation patterns:

- The post-sunset at low latitudes is characterized by low $\mid<$ ROT $>\mid$ and large ROT_SD, which indicates the simultaneous presence of several scale sizes, either below or above the Fresnel scale. This configuration leads to both amplitude and phase scintillation, as shown in the percentage occurrence maps.

- The same pattern of low latitude post-sunset (low $\mid<$ ROT $>\mid$ and large ROT_SD) is found in a narrow latitudinal band at around $-23^{\circ} \mathrm{N}$, which is characterized by small dependence on time. This is explained in terms of the same scale sizes active for scintillation, but here concentrated in a well-defined Mlat range. This independence might indicate a source of scintillation that is independent of the daily variability. The most likely source is the particle flux in the SAMA.

- The high latitude is characterized by phase scintillation only, which follows the patterns of enhanced ROT_SD quite closely. These patterns mainly highlight three regions: the cusp, the post-midnight cap, and the boundaries of the auroral oval (mainly around magnetic midnight). The $<$ ROT $>$ /ROT_SD configuration here indicates the possible presence of structures of all scales, but the lack of amplitude scintillation suggests the absence of structures with scales below the Frensel zone.

In this study, the ionospheric scintillation hot-spot analysis is also introduced and used to derive the seasonal variations of the scintillation parameters in specific areas of the ionosphere in which the probability of signal 
degradation due to scintillation is large. The hot spots are identified through the Mlat and MLT ranges, and are termed as: POST, SAMA, CUSP, TAIL and PATC. This analysis mainly shows the very different conditions of the ROT parameters at high and low latitudes in driving scintillation, and provides evidence of the seasonality induced by equinoxes and the austral summer.

The GBSC is now a mature and robust technique that provides significant indications of the spatial/ temporal recurrences of plasma irregularities. The complementary use of the GBSC and of the hot-spot analysis is useful to extend our current knowledge of the mechanisms that cause scintillations. An extension of the network and the future inclusion of different data sources in the GBSC would improve the coverage of the maps in the 'transition regions' between the mid to high latitudes and the mid to low latitudes, and (at least partially) improve our understanding of the stillopen scientific questions that have emerged from the study presented here.

Acknowledgements. This study is part of the activities of the GRAPE Expert group (www.grape.scar.org), funded by SCAR, within the framework of which a fruitful and close collaboration was born among different groups operating scintillation monitors. LS, LA, GDF and VR thank the Programma Nazionale di Ricerche in Antartide (PNRA, Italian National Program for Antarctic Research). PC thanks the South African National Antarctic Programme (SANAP grant SNA2011120100008) for logistic support. EC thanks the CNPq/PROANTAR (proc. 0520186/06-0 and 556872/2009-6), INCT-APA (Instituto Nacional de Ciência e Tecnologia Antártico de Pesquisas Ambientais, CNPq proc. 574018/2008-5 and FAPERJ proc. E-16/170.023/2008) and the SECIRM and Brazilian Antarctic Program (PROANTAR). The authors also thank the SCAR, the National Space Science Data Center (NSSDC) for the software calculating the auroral oval position and the authors of the model (Feldstein, Holzworth and Meng).

\section{References}

Abdu, M.A., I.S. Batista, A.J. Carrasco and C.G.M. Brum (2005). South Atlantic magnetic anomaly ionization: a review and a new focus on electrodynamic effects in the equatorial ionosphere, J. Atm. Sol. Terr. Phys., 67, 1643-1657.

Abdu, M.A., E. Alam Kherani, I.S. Batista, E.R. de Paula, D.C. Fritts and J.H.A. Sobral (2009). Gravity wave initiation of equatorial spread $\mathrm{F}$ / plasma bubble irregularities based on observational data from the SpreadFEx campaign, Annales Geophysicae, 27, 2607-2622; doi:10.5194/angeo-27-2607-2009.

Alfonsi, L., L. Spogli, G. De Franceschi, V. Romano, M. Aquino, A. Dodson and C.N. Mitchell (2011). Bipolar climatology of GPS ionospheric scintillation at solar minimum, Radio Sci., 46, RS0D05; doi:10.102 9/2010RS004571.

Baker, K.B., and Wing, S. (1989). A new magnetic co- ordinate system for conjugate studies at high latitudes, J. Geophys. Res., 94, 9139-9143.

Basu, S., K.M. Groves, S. Basu and P.J. Sultan (2002). Specification and forecasting of scintillations in communication/navigation links: current status and future plans, J. Atmos. Sol. Terr. Phys., 64, 1745-1754.

Cabrera, M.A., M. Pezzopane, E. Zuccheretti and R.G. Ezquer (2010). Satellite traces, range spread-F occurrence, and gravity wave propagation at the southern anomaly crest, Annales Geophysicae, 28, 1133-1140.

Committee on the Societal and Economic Impacts of Severe Space Weather Events (200). Severe Space Weather Events - Understanding Societal and Economic Impacts Workshop Report, ISBN 0-30912770-X.

De Franceschi, G., L. Alfonsi, V. Romano, M.H.O. Aquino, A. Dodson, C.N. Mitchell and A.W. Wernik (2008). Dynamics of high latitude patches and associated small scale irregularities, J. Atmos. Sol.-Terr. Phys., 70, 879-888; doi:10.1016/j.jastp.2007.05.018.

Fejer, B.G., E.R. de Paula, I.S. Batista and R.F. Woodman (1989). Equatorial F region vertical plasma drifts during solar maxima, J. Geophys. Res., 94, 12049-12054.

Fejer, B.G., E.R. de Paula, S.A. Gonzalez and R.F. Woodman (1991). Average vertical and zonal F region plasma drifts over Jicamarca, J. Geophys. Res., 96, 13901-13906.

Feldstein, Y.I. (1963). On morphology and auroral and magnetic disturbances at high latitudes Geomagn. Aeron., 3, 183-192.

Fisher, G., and J. Kunches (2011). Building resilience of the global positioning system to space weather, Space Weather, 9, S12004; doi:10.1029/2011SW000718.

Holzworth, R.H., and C.-I. Meng (1975). Mathematical representation of the auroral oval, Geophys. Res. Lett., 2, 377-380.

Jin, S.G., O. Luo, P. Park (2008). GPS observations of the ionospheric F2-layer behavior during the $20^{\text {th }}$ November 2003 geomagnetic storm over South Korea. J. Geod. 82, 883-892; doi:10.1007/ s00190-0080217-x.

Kintner, P.M., T. Humphreys and J. Hinks (2009). GNSS and Ionospheric Scintillation - How to survive to the next solar maximum, InsideGNSS, July / August 2009, 22-30.

Mannucci, A.J., B.D. Wilson and C.D. Edwards (1993). A new method for monitoring the Earth ionosphere total electron content using the GPS global network, Paper presented at ION GPS 93, Inst. of Navig., Salt Lake City, Utah.

Rino, C.L. (1979a). A power law phase screen model for 
ionospheric scintillation: 1. Weak scatter, Radio Sci., 14 (6), 1135-1145.

Rino, C.L. (1979b). A power law phase screen model for ionospheric scintillation: 2 . Strong scatter, Radio Sci., 14 (6), 1147-1155.

Spogli, L., L. Alfonsi, G. De Franceschi, V. Romano, M.H.O. Aquino and A. Dodson (2009). Climatology of GPS ionospheric scintillations over high and midlatitude European regions, Annales Geophysicae, 27, 3429-3437.

Spogli, L., L. Alfonsi, G. De Franceschi, V. Romano, M.H.O. Aquino and A. Dodson (2010). Climatology of GNSS ionospheric scintillations at high and mid latitudes under different solar activity conditions, Il Nuovo Cimento B, 5/ 6, 623-632; doi:10.1393/ncb/ i2010-10857-7.

Taylor, J.R. (1997). An Introduction to Error Analysis: The Study of Uncertainties in Physical Measurement, $2^{\text {nd }}$ ed., Univ. Sci., Sausalito, California.

Tsunoda, R.T. (1988). High-Latitude F region irregularities: a review and synthesis, Rev. Geophys., 26, 719-760.

Van Dierendonck, A.J., J. Klobuchar and Q. Hua (1993). Ionospheric scintillation monitoring using commercial single frequency C/A code receivers, In: ION GPS-93 Proceedings of the Sixth International Technical Meeting of the Satellite Division of the Institute of Navigation, (Salt Lake City, U.S.A., September 22-24, 1993), 1333-1342.

Wernik, A.W., J.A. Secan and E.J. Fremouw (2003). Ionospheric irregularities and scintillation, Adv. Space Res., 31 (4), 971-981.

Yeh, K.C., and C.H. Liu (1982). Radio wave scintillations in the ionosphere, Proceedings of the IEEE 70 (4), 324-360.

Young, E.R., W. J. Burke, F.J. Rich and R.C. Sagalyn (1984). The distribution of topside spread $\mathrm{F}$ from in situ measurements by Defense Meteorological Satellite Program: F2 and F4, J. Geophys. Res., 89, 5565-5573.

\footnotetext{
${ }^{\star}$ Corresponding author: Luca Spogli,

Istituto Nazionale di Geofisica e Vulcanologia, Sezione Roma 2,

Rome, Italy; email: luca.spogli@ingv.it.

C 2013 by the Istituto Nazionale di Geofisica e Vulcanologia. All

rights reserved.
} 